\title{
Armazenamento de produtos para a saúde estéreis, sistemas de barreira e
}

\section{sustentabilidade do meio ambiente}

\author{
Storage of sterile health products, barrier systems and environmental sustainability \\ Almacenamiento de productos para la salud esteril, sistemas de barreira y sustentabilidad del \\ medio ambiente
}

\section{Resumo}

Objetivo: analisar as produções cientificas sobre armazenamento de produtos para a saúde estéreis, repercussões para a CME e sustentabilidade do meio ambiente. Método: Revisão integrativa da literatura, estratégia PICo e procedimentos definidos pelo Preferred Reporting Items for Systematic Reviews and Meta-Analyses (PRISMA) para a seguinte questão norteadora: Quais as implicações do armazenamento de produtos para a saúde estéreis para a CME e sustentabilidade do meio ambiente? Buscas realizadas entre abril e junho de 2021, sem definição de tempo. Idioma dos estudos: português, inglês e espanhol. Resultados: Estudos brasileiros (66,7\%). Os demais eram provenientes da China, Indonésia, Índia e Estados Unidos. O ano de 2015 foi o mais prevalente (33,3\%). Estudos descritivos (53,3\%) e experimentais (20\%). Conclusão: Área de armazenamento requer controle da umidade, temperatura e da descontaminação. Falhas como pacotes incompletos, produtos vencidos, resíduos orgânicos pós-esterilização, materiais limpos e sujos no armazenamento exigiram novo processamento. O uso do não tecido reduziu taxa de resterilização. 
Identificado o complexo processo de reprocessamento de campos de tecido de algodão. A necessidade de educação continuada permitirá a revisão de práticas incorretas e o conhecimento de recomendações dos órgãos regulamentadores que precisam ser atendidas.

Palavras-chave: Esterilização; Armazenamento; Embalagem de produtos.

\begin{abstract}
Objective: To analyze scientific productions about storage of sterile health products, repercussions to the CSSD and environmental sustainability. Method: Integrative literature review, PICo strategy and defined procedures by Preferred Reporting Items for Systematic Reviews and Meta-Analyses (PRISMA) base on the following question: What are the implications of storing sterile health products to the CSSD and the environmental sustainability? Researches carried out from April to June 2021, without defined time, in Portuguese, English and Spanish. Results: studies from Brazil $(66,7 \%)$. The others were from China, Indonesia, India and United States of America. The year of 2015 was the most prevalent $(33,3 \%)$. Descriptive $(53,3 \%)$ and experimental $(20 \%)$ studies. Conclusion: Storage area requires humidity control, temperature and disinfection. Failures such as incomplete packages, products with expired date, organic wastes after sterilization, clean and dirty materials in the storage required reprocessing. The use of the SMS reduced the rate of resterilization. It was identified the complex reprocessing of the drapes made of cotton. The need for continuing education will enable the review of bad practices as well as the knowledge of recommendations from regulatory agencies that need to be attended.
\end{abstract}

Keywords: Sterilization; Storage; Product packing.

\title{
Resumen
}

Objetivo: analisar las producciones cientificas al respecto del almacenamiento para la salud estéril, repercusiones para la CME y sustentabilidade del medio ambiente. Método: Revisión integradora de la literatura estrategica PICo y procedimientos definidos por el Preferred Reporting Items for Systematic Reviews and Meta-Analyses (PRISMA) para la seguiente pregunta guía: cuales son las implicaciones del almacenamiento de produtos para la salud estéril para la CME y sustentabilidad del medio ambiente? Búsquedas hechas entre abril y junio de 2021, sin definiciones de tiempo. Idiomas del estúdio: portugués, inglés y español. Resultados: Estudios Brasileños $(66,7 \%)$. Los demás fueron de la China, Indonesia, Índia y Estados Unidos. El año de 2015 fue el más frecuente (33,3\%). Estudios descriptivos (53,3\%) y experimentales (20\%). Conclusión: área de almacenamiento requiere control de humidad, temperatura y descontaminación. Fallas como paquetes incompletos, productos caducados, desechos orgânicos posteriores a la esterilización, materiales limpios y sucios em el almacenamiento requerieron um nuevo procesamiento. El uso de tela no tejida reduce la tasa de reesterilización. Identificado el complejo proceso de reprocesamiento de los campos de tejidos de algodón. La necesidad de la educación contínua permitirá la revisión de practicas incorrectas y el conocimiento de recomendaciones de los organismos reguladores que deben cumplirse.

Palabras clave: Esterilización; Almacenamiento; Empaquetado del producto.

\section{Introdução}

Este estudo trata do armazenamento de produtos para a saúde esterilizados, que constitui uma das fases do processamento de produtos realizada na unidade de Central de Material e Esterilização (CME).

A CME é a unidade funcional destinada ao processamento de produtos para saúde. Esse inclui um conjunto de ações relacionadas tais como a pré-limpeza, recepção, limpeza, secagem, avaliação da integridade e da funcionalidade, preparo, desinfecção ou esterilização, armazenamento e distribuição para as unidades consumidoras. O preparo e armazenamento foram abordados de forma mais aprofundada nesse estudo incluindo ainda os sistemas de barreira estéril (SBE) e a sustentabilidade do meio ambiente. A RDC $\mathrm{n}^{\circ} 15 / 2012$ dispõe sobre requisitos de boas práticas para o processamento de produtos para saúde e constitui a regulamentação vigente no Brasil (Brasil, 2012).

As embalagens, atualmente denominadas sistemas de barreira estéril, são insumos utilizados na fase de preparo dos produtos para a saúde tem como função permitir a esterilização do conteúdo, protegendo-o de possíveis contaminações até que seja utilizado, permitindo a apresentação asséptica (SOBECC, 2013).

Os SBE, para que sejam considerados ideais, devem minimamente possuir as seguintes características: permitir adequada penetração e remoção do agente esterilizante; constituir barreira para os microrganismos; resistir a rasgos, abrasões e perfurações; permitir selagem eficiente e que permita perceber violação; proporcionar abertura asséptica e não delaminar; ser atóxicas, inodoras e não liberar corantes; ser isentas de rasgos, furos, fissuras, dobras ou espessura reduzida localizada; ter 
nível aceitável de limpeza se processáveis; não liberar partículas; ter bom custo-benefício; garantir proteção durante o manuseio; repelir umidade; quando possível, ter indicador químico de processo impregnado; estar disponível em vários tamanhos; ter baixa memória, facilitando a abertura dos pacotes; ser fácil de manipular durante o preparo e ser apropriada ao método de esterilização escolhido (AORN, 2017).

Os sistemas de barreira estéril descartáveis incluem o papel grau cirúrgico, SMS (spunbonded/meltblown/spunbonded), papel crepado e o Tyvek. Os reaproveitáveis incluem o tecido de algodão e contêineres rígidos. Todos esses sistemas são considerados embalagens primárias. Outros insumos de acondicionamento incluem as coverbags, coberturas plásticas secundárias que tem como objetivo proteger a embalagem primária de perfurações, abrasões, poeira e da manipulação excessiva (Oliveira \& Silva, 2015).

Os sistemas de barreira estéril descartáveis ou reaproveitáveis trazem implicações sobre o meio ambiente. O estudo da percepção dos riscos inerentes aos resíduos gerados nos estabelecimentos de assistência à saúde é relevante para a elaboração e a implementação de ações que minimizem danos aos trabalhadores, à comunidade e ao ambiente (Justiniano, Eduardo, Binotto, Macedo, Veiga, Tognoli, \& Mendes, 2020).

Os itens utilizados no acondicionamento de PPS estéreis, após o uso, são responsáveis pela geração considerável de resíduos sólidos. Estes têm preocupado os gestores de saúde, porque os modelos de gestão devem pautar suas decisões na responsabilidade ambiental e social para o desenvolvimento econômico. Isso implica na criação de políticas públicas e legislações com eixos de orientação relacionados à sustentabilidade do meio ambiente e à preservação da saúde (Nogueira, Santos, Cardelli, \& Castilho, 2020).

O desafio para estabelecimento de práticas sustentáveis nos hospitais encontra como importante desafio a relação custo benefício. Apesar dos avanços tecnológicos na fabricação de produtos para a saúde de uso único, os produtos reutilizáveis equivalentes, ainda são mais custo-efetivos, apesar do custo de processamento na CME (Pereira, Pascoal, Rolim, Ferreira, \& Silva, 2020).

A justificativa para este estudo se apoia na necessidade atual de empreender pesquisas e iniciativas que defendam o planeta, otimizem a utilização de recursos naturais como a água; que tratem da segregação, destinação de resíduos sólidos. Nas últimas décadas, instrumentos legais e normativos têm sido desenvolvidos para regulamentar o controle da poluição ambiental e fomentar a adoção de sistemas de gestão ambiental no setor de saúde (Oliveira, Viana, \& Castañon, 2018).

Essa aproximação entre saúde e sustentabilidade do meio ambiente será fundamental para o futuro do mundo.

Os sistemas de barreira estéril (SBE) constituem o objeto desse estudo que abordará esses insumos, a área de armazenamento e os impactos sobre a sustentabilidade do meio ambiente.

Nesse contexto, apresenta-se a questão de pesquisa: Quais as implicações do armazenamento de produtos para a saúde estéreis para a CME e sustentabilidade do meio ambiente?

O estudo tem por objetivo analisar as produções cientificas sobre armazenamento de produtos para a saúde estéreis, repercussões para a CME e sustentabilidade do meio ambiente.

\section{Metodologia}

Estudo de revisão integrativa da literatura, com a finalidade de agregar e condensar estudos relativos ao tema investigado. Esse tipo de revisão descreve o conhecimento atual sobre uma temática específica, pois é guiado de modo a identificar, analisar e sintetizar resultados de publicações autônomas sobre o mesmo assunto (Joanna Briggs Institute, 2014).

A partir da pergunta de pesquisa foram organizados os termos para busca segundo o acrônimo de revisões integrativas - PICo P (População), I (Fenômeno de interesse) e Contexto (Co). Onde P - Produtos para a saúde esterilizados, I armazenamento Co - Centro de Material e Esterilização (CME). 
A identificação dos termos nos vocabulários controlados Descritores em Ciências da Saúde (DECS), Medical Subject Healding (MESH) e Emtree (Embase subject headings) e na busca preliminar. Estes foram organizados com os operadores booleanos $O R$ - permite o agrupamento/soma dos sinônimos $e$ AND - faz a interseção dos termos. O operador NOT tem a função de exclusão - não foi utilizada nesta pesquisa, como apresentados no Quadro 1.

Quadro 1 - Termos identificados nos vocabulários controlados, Rio de Janeiro 2021.

\begin{tabular}{|c|c|}
\hline PICo & MAPEAMENTO \\
\hline \multirow{2}{*}{ 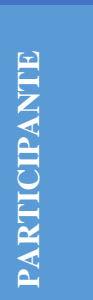 } & $\begin{array}{l}\text { "Embalagem de Equipamentos e Provisões" OR "Embalaje de Equipos y Suministros" OR armazenamento OR armazenagem } \\
\text { OR armazenar OR almacenar OR "Embalagem de Produtos" OR Embalagem OR "Recipientes de Produtos" OR "Embalaje de } \\
\text { Productos" OR "Contenedores de Productos" OR "Empaque de Productos" OR Emballage }\end{array}$ \\
\hline & $\begin{array}{l}\text { "Equipment and Supplies Packing" OR "Packing of Equipment and Supplies" OR "Sterilization packaging" OR "sterile } \\
\text { packaging" OR "Sterile packaging" OR "sterile barrier" OR "microbial barrier" OR "Product Packaging" OR "Product } \\
\text { Packagings" OR Packaging* OR "Product Containers" OR "Product Container" }\end{array}$ \\
\hline \multirow{2}{*}{ 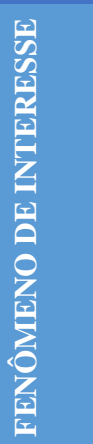 } & $\begin{array}{l}\text { "Equipamentos e Provisões" OR "Aparelhos e Instrumentos" OR Dispositivo* OR "Dispositivo Médico" OR "Dispositivos } \\
\text { Médicos" OR Equipamento* OR Inventário* OR Provisões OR "Equipos y Suministros" OR "Aparatos e Instrumentos" OR } \\
\text { Equipo* OR Stocks OR "Equipamentos e Provisões Hospitalares" OR "Equipos y Suministros de Hospitales" OR "Produto } \\
\text { Médico-Hospitalar" OR "Produtos Médico-Hospitalares" OR "Produtos Médicos Hospitalares" OR "Equipo y Suministro de } \\
\text { Hospitales" OR "processamento de produtos para a saúde" OR "procesamiento para la salud" OR "produtos para a saúde" }\end{array}$ \\
\hline & $\begin{array}{l}\text { "Equipment and Supplies" OR "Apparatus and Instruments" OR Device* OR Equipment OR "Instruments and Apparatus" OR } \\
\text { Inventories OR Inventory OR "Medical Device" OR "Medical Devices" OR Supplies OR "Supplies and Equipment" OR } \\
\text { "Equipment and Supplies, Hospital" OR "Hospital Equipment and Supplies" OR "Hospital Supplies" OR "Hospital Supply" } \\
\text { OR "Hospital Equipment" OR "processing of health products" OR "health product processing" }\end{array}$ \\
\hline \multirow{2}{*}{ 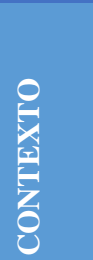 } & $\begin{array}{l}\text { Esterilização OR "Centro de Esterilização" OR Esterilización OR "Centro de Material e Esterilização" OR CME OR "Central de } \\
\text { esterilização" OR "centro de material y esterilización" OR Desinfecção OR Desinfección OR "central de material e } \\
\text { esterilização" OR "Central de Material de Esterilização" }\end{array}$ \\
\hline & $\begin{array}{l}\text { Sterilization OR Disinfection OR "central sterile supply department" OR " Sterile Processing Department" OR "central sterile } \\
\text { supply" OR "center of material and sterilization" OR "Sterilization Central Supply" }\end{array}$ \\
\hline
\end{tabular}

Fonte: Autores (2021).

As buscas foram realizadas em junho de 2021 nas bases de dados referenciais e literatura cinzenta: Portal Regional da Biblioteca Virtual em Saúde (BVS) e nas suas principais bases de dados - Literatura Latino-Americana e do Caribe em Ciências da Saúde (LILACS), Bibliográfico Español em Ciências (IBECS), Coleção Nacional das Fontes de Informação do SUS - ColecionaSUS dentre outras. No Portal Pubmed da National Library of Medicine (NLM) e Scientific Electronic Library Online (Scielo).

No Portal de Periódicos da Capes foram empregadas as bases de dados: Elsevier: Embase e Scopus, Clarivate Analytics: Web of Science e Ebsco: Academic Search Premier (ASP) e Cummulative Index to Nursing and Allied Health Literature (CINAHL).

Foram selecionados critérios de inclusão: artigos publicados em português, inglês e espanhol, disponíveis online, gratuitos e completos. E como critérios de exclusão: os repetidos, os não disponíveis na íntegra, publicações manuscritas como revisão de literatura, caso clinico, tese e dissertações. Não foi utilizado como critério de elegibilidade a época das publicações, tendo em vista a necessidade da análise abrangente relacionada ao tema, porém, deu-se prioridade a publicações mais recentes. 
Após identificação das duplicações, os resultados das buscas foram exportados para o aplicativo Rayyan do Qatar Computing Research Institute, QCRI onde foi realizado o processo de seleção de título e resumo, o estudo seguiu as etapas de elaboração recomendadas pelo (Preferred Reporting Items for Systematic Reviews and Meta-Analyses (PRISMA) (Galvão, Pansani, \& Harrad, 2015), como demonstra a Figura 1.

Figura 1 - Fluxograma do processo de busca e seleção, Rio de Janeiro.

\section{Identificação de estudos por meio de bancos de dados e registros de seleção}

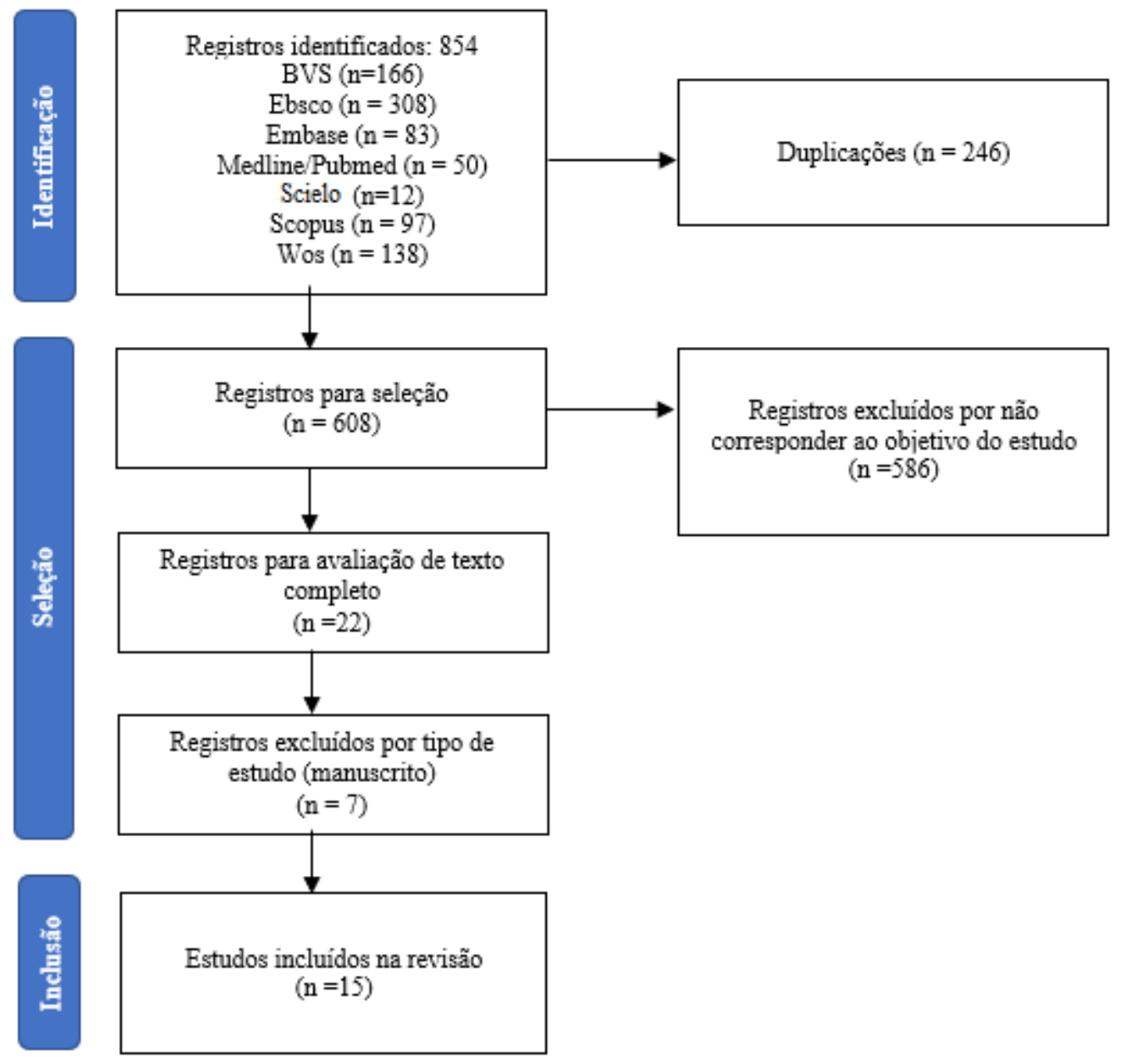

Fonte: Autores (2021)

\section{Resultados}

O Quadro 2 caracteriza os estudos, apresentando área temática, ano, autores, periódico e síntese dos resultados. 
Quadro 2 - Caracterização dos estudos que compuseram a amostra, segundo título, autores, ano, país, periódicos, tipo de estudo e síntese dos resultados, Rio de Janeiro 2021.

\begin{tabular}{|c|c|c|c|}
\hline & $\begin{array}{c}\text { Título } \\
\text { Autores }\end{array}$ & $\begin{array}{c}\text { Ano/País/Periódico/Tipo } \\
\text { de estudo }\end{array}$ & Síntese dos resultados \\
\hline 01 & $\begin{array}{l}\text { "ÁREA DE ARMAZENAMENTO } \\
\text { DE PRODUTOS PARA SAÚDE: } \\
\text { REPENSANDO A FREQUÊNCIA } \\
\text { DA DESCONTAMINAÇÃO DE } \\
\text { PRATELEIRAS" } \\
\text { Tipple, dos Santos, de Melo Costa, } \\
\text { Bouwman, \& Goulart. }\end{array}$ & $\begin{array}{c}2020 \\
\text { Brasil } \\
\text { CIENC CUID SAUDE } \\
\text { Estudo transversal, } \\
\text { descritivo }\end{array}$ & $\begin{array}{l}\text { Objetivo: avaliar o risco potencial para ocorrência de } \\
\text { eventos relacionados pela descontaminação de prateleiras } \\
\text { da área de armazenamento e distribuição. Esses eventos } \\
\text { incluíram toque excessivo no sistema de barreira, } \\
\text { manuseio incorreto do produto ou pela falta de } \\
\text { higienização das mãos. A frequência de descontaminação } \\
\text { dessa área limpa foi questionada face ao risco para a } \\
\text { manutenção da esterilidade dos produtos. }\end{array}$ \\
\hline 02 & $\begin{array}{l}\text { “CONDIÇÕES DE TEMPERATURA } \\
\text { E UMIDADE NA SALA DE } \\
\text { DISTRIBUIÇÃO E } \\
\text { ARMAZENAMENTO DOS } \\
\text { PRODUTOS PARA SAÚDE” } \\
\text { Nunes, Fassarella, Souza, Meneses, } \\
\text { Silva, \& Luna. }\end{array}$ & $\begin{array}{c}2019 \\
\text { Brasil } \\
\text { REV. ENFERMAGEM } \\
\text { ATUAL IN DERME } \\
\text { Estudo retrospectivo } \\
\text { quantitativo }\end{array}$ & $\begin{array}{l}\text { Objetivo: identificar as condições ambientais, de } \\
\text { temperatura e umidade, na área de armazenamento e } \\
\text { distribuição de produtos para saúde esterilizados. Ambas } \\
\text { as condições ambientais, de temperatura e de umidade, } \\
\text { exibiram resultados em sua maioria, fora dos padrões } \\
\text { recomendados por sociedades de especialistas nacionais e } \\
\text { internacionais (SOBECC e AORN), }\end{array}$ \\
\hline $\mathbf{0 3}$ & $\begin{array}{l}\text { "ERRORS IN PACKAGING } \\
\text { SURGICAL INSTRUMENTS } \\
\text { BASED ON A SURGICAL } \\
\text { INSTRUMENT TRACKING } \\
\text { SYSTEM: AN OBSERVATIONAL } \\
\text { STUD” } \\
\text { Zhu, Yuan, Li, \& Cheng. }\end{array}$ & $\begin{array}{c}2019 \\
\text { China } \\
\text { BMC HEALTH } \\
\text { SERVICES RESEARCH } \\
\text { Estudo diagnóstico }\end{array}$ & $\begin{array}{l}\text { Objetivo: investigar erros no processamento de } \\
\text { instrumentos cirúrgicos. } 33.839 \text { pacotes examinados. } \\
\text { Erros mais frequentes: especificação errada }(44 \%) \text {, falta } \\
\text { de instrumentos }(19,4 \%) \text {; pacote incompleto }(17,6 \%) \text {. } \\
\text { Erros mais frequentes entre enfermeiros com até } 1 \text { ano de } \\
\text { experiência }(2,04 \%) \text {. Maior taxa de pacotes com erros } \\
\text { produzidos no horário entre } 16 \text { e } 22 \text { horas }(1,35 \%)\end{array}$ \\
\hline 04 & $\begin{array}{l}\text { "PRAZO DE VALIDADE DE } \\
\text { ESTERILIZAÇÃO DE ARTIGOS } \\
\text { UTILIZADOS EM UMA CLINICA } \\
\text { ODONTOLÓGICA” } \\
\text { Maciel, Martins, Mendes, Kozusny- } \\
\text { Andreani, \& de Souza. }\end{array}$ & $\begin{array}{c}2019 \\
\text { Brasil } \\
\text { REVISTA NURSING } \\
\text { Estudo experimental }\end{array}$ & $\begin{array}{l}\text { Objetivos: avaliar a manutenção da esterilidade de } \\
\text { materiais armazenados em uma clínica escola de } \\
\text { odontologia. Constatada ocorrência de UFC nos grupos e } \\
\text { amostras avaliados. Seis tipos de microrganismos } \\
\text { avaliados. Os artigos mantiveram esterilidade por } 84 \text { dias } \\
\text { de prateleira. Sugere-se o armazenamento em armários } \\
\text { fechados com portas, manter o controle de temperatura e } \\
\text { umidade relativa do ambiente e manter uma limpeza } \\
\text { diária. }\end{array}$ \\
\hline 05 & $\begin{array}{l}\text { "LOOKING FOR HOLES IN } \\
\text { STERILE WRAPPING: HOW } \\
\text { ACCURATE ARE WE?" } \\
\text { Rashidifard, Mayassi, Bush, Opalacz, } \\
\text { Richardson, Muccino, \& DiPasquale. }\end{array}$ & $\begin{array}{c}2018 \\
\text { Estados Unidos } \\
\text { CLINICAL } \\
\text { ORTHOPAEDICS AND } \\
\text { RELATED RESEARCH } \\
\text { Estudo diagnóstico }\end{array}$ & $\begin{array}{l}\text { Objetivo: determinar a frequência de furos em pacotes } \\
\text { esterilizados e as diferenças relacionadas aos diferentes } \\
\text { observadores. } 30 \text { profissionais. } 2 \text { hospitais. Diferentes } \\
\text { tipos de iluminação. } 46 \text { pacotes examinados por cada } \\
\text { profissional. Orifícios menores que } 2 \mathrm{~mm} \text { não foram } \\
\text { detectados. O tempo de experiência profissional, o tipo de } \\
\text { iluminação e o tempo de inspeção não interferiram nos } \\
\text { resultados. Estudo de simulação. }\end{array}$ \\
\hline 06 & $\begin{array}{l}\text { “CAUSAS DE RETRABALHO DE } \\
\text { PRODUTOS PARA SAÚDE NO } \\
\text { CENTRO DE MATERIAIS E } \\
\text { ESTERILIZAÇÃO” } \\
\text { Alvim, \& de Souza. }\end{array}$ & $\begin{array}{c}2018 \\
\text { Brasil } \\
\text { REV. SOBECC } \\
\text { Estudo descritivo } \\
\text { quantitativo }\end{array}$ & $\begin{array}{l}\text { Objetivo: identificar as principais causas de retrabalho de } \\
\text { produtos para saúde (PPS) em uma CME. Foram } \\
\text { encontrados } 605 \text { itens de retrabalho, que representaram } \\
\text { uma taxa de } 0,75 \% \text { do total de caixas e pacotes } \\
\text { produzidos. As principais causas foram relacionadas a } \\
\text { produtos vencidos ( } 74 \% \text { ) e resíduos orgânicos pós- } \\
\text { esterilização (13\%). }\end{array}$ \\
\hline 07 & $\begin{array}{l}\text { “ARMAZENAMENTO DOS } \\
\text { PRODUTOS PARA SAÚDE EM } \\
\text { CENTROS DE ESTERILIZAÇÃO } \\
\text { DE HOSPITAIS” } \\
\text { Mussel, de Paula, \& de Oliveira. }\end{array}$ & $\begin{array}{c}2017 \\
\text { Brasil } \\
\text { ENFERM. FOCO } \\
\text { Estudo quantitativo }\end{array}$ & $\begin{array}{l}\text { Objetivo: descrever condições de armazenamento dos } \\
\text { produtos estéreis em Centros de Material Esterilizado de } \\
\text { hospitais de grande porte. O acondicionamento do } \\
\text { produto para saúde em armários fechados (60\%), } \\
\text { revestimento lavável ( } 100 \%) \text {, com controle de umidade } \\
\text { relativa do ar e temperatura. Controle da data de validade } \\
\text { dos produtos (100\%); transporte em carros exclusivos em } \\
80 \% \text {; } 30 \% \text { não possuíam área física exclusiva para }\end{array}$ \\
\hline
\end{tabular}




\begin{tabular}{|c|c|c|c|}
\hline & & & $\begin{array}{l}\text { produtos estéreis. Falhas no cumprimento da legislação } \\
\text { vigente: materiais limpos e sujos no setor de } \\
\text { armazenamento; prateleira aberta, incidência de luz solar } \\
\text { direta, falta de controle da umidade e temperatura, e } \\
\text { tubulações expostas. }\end{array}$ \\
\hline 08 & $\begin{array}{l}\text { "THE STERILITY OF REUSABLE } \\
\text { INSTRUMENTS AT CENTRAL } \\
\text { SURGERY INSTALLATION'S } \\
\text { ROOM STORAGE OF ONE OF THE } \\
\text { HOSPITAL IN BANDUNG" } \\
\text { Kurniawansyah, \& Mita. }\end{array}$ & $\begin{array}{c}2017 \\
\text { Indonésia } \\
\text { J. PHARM. SCI. \& RES } \\
\text { Estudo laboratorial }\end{array}$ & $\begin{array}{l}\text { Objetivo: determinar o efeito do tempo de estocagem } \\
\text { sobre a condição de esterilidade de instrumentos na área } \\
\text { de armazenamento. Hospital em Bandung, China. No } 12^{\circ} \text {, } \\
16^{\circ} \text { e } 17^{\circ} \text { dias de armazenamento foram identificados } \\
\text { instrumentos não estéreis na área de armazenamento. } \\
\text { Fatores ambientais e relacionados aos profissionais foram } \\
\text { atribuídos como responsáveis por essa contaminação. }\end{array}$ \\
\hline 09 & $\begin{array}{l}\text { "SEGURANÇA DO PACIENTE: } \\
\text { EMBALAGENS, } \\
\text { ACONDICIONAMENTO E TEMPO } \\
\text { DE GUARDA DE MATERIAIS } \\
\text { ESTERILIZADOS NA ATENÇÃO } \\
\text { BÁSICA" } \\
\text { Reisdorfer, de Araujo, da Silva, dos } \\
\text { Santos, \& Soder. }\end{array}$ & $\begin{array}{c}2016 \\
\text { Brasil } \\
\text { Araújo GM et al } \\
\text { CIENC CUID SAUDE } \\
\text { Estudo exploratório } \\
\text { qualitativo }\end{array}$ & $\begin{array}{l}\text { Objetivo: compreender como enfermeiros, técnicos, } \\
\text { auxiliares em enfermagem e auxiliares de consultório } \\
\text { dentário procedem no cuidado com embalagens, } \\
\text { acondicionamento e tempo de guarda de materiais } \\
\text { esterilizados nas unidades de atenção básica. Identificadas } \\
\text { necessidades: tipo de embalagem, acondicionamento } \\
\text { produtos críticos. Evidenciada necessidade de capacitação } \\
\text { contínua dos profissionais para padronização dos serviços } \\
\text { nas CMEs de acordo com normas vigentes. }\end{array}$ \\
\hline 10 & $\begin{array}{l}\text { "IS NON-WOVEN FABRIC A } \\
\text { USEFUL METHOD OF PACKING } \\
\text { INSTRUMENTS FOR OPERATING } \\
\text { THEATRES IN RESOURCE } \\
\text { CONSTRAINED SETTINGS?" } \\
\text { Devadiga, Thomas, Shetty, \& Setia. }\end{array}$ & $\begin{array}{c}2015 \\
\text { Índia } \\
\text { INDIAN JOURNAL OF } \\
\text { MEDICAL } \\
\text { MICROBIOLOGY } \\
\text { Estudo retrospectivo }\end{array}$ & $\begin{array}{l}\text { Objetivo: Avaliar a proporção da resterilização após a } \\
\text { introdução do não tecido para empacotamento de } \\
\text { instrumentos cirúrgicos e a avaliação custo benefício do } \\
\text { uso desse sistema de barreira. Período } 2019 \text { a } 2013 \text {. } \\
\text { Análise de } 117.335 \text { pacotes. Redução de taxa de } \\
\text { resterilização de } 9 \text { para } 0,5 \% \text { nesse período de } 4 \text { anos } \\
\text { após a introdução do não tecido. }\end{array}$ \\
\hline 11 & $\begin{array}{l}\text { “RELATIONSHIP BETWEEN } \\
\text { TEMPERATURE AND HUMIDITY } \\
\text { ON STERILITY OF REUSABLE } \\
\text { INSTRUMENTS IN HOSPITAL’S } \\
\text { CSSD” } \\
\text { Kurniawansyah, Abdassah, \& } \\
\text { Gondodiputro. }\end{array}$ & $\begin{array}{c}2015 \\
\text { Indonesia } \\
\text { INT. J. PHARM. SCI. } \\
\text { REV. RES } \\
\text { Estudo exploratório }\end{array}$ & $\begin{array}{l}\text { Objetivo: analisar a relação entre temperatura e umidade } \\
\text { da área de armazenamento de uma CME. Temperaturas } \\
\text { na área de armazenamento: entre } 25,3 \text { e } 27,2^{\circ} \mathrm{C} \text {. Umidade } \\
\text { variando entre } 47 \text { e } 57 \% \text {. Confirmada relação entre } \\
\text { temperatura e umidade para a manutenção da esterilidade } \\
\text { na área de estocagem: baixas temperaturas, baixa } \\
\text { umidade. Causas prováveis para contaminação na área de } \\
\text { estocagem: condicionamento de ar, descontaminação, } \\
\text { empacotamento, estocagem e monitoramento do produto } \\
\text { final. }\end{array}$ \\
\hline 12 & $\begin{array}{l}\text { “(DES)CUIDADO COM } \\
\text { PRODUTOS PARA SAÚDE } \\
\text { PROCESSADOS NO } \\
\text { TRANSPORTE E } \\
\text { ARMAZENAMENTO EM } \\
\text { UNIDADES DE INTERNAÇÃO” } \\
\text { Freitas, Tipple, Pires, Melo, \& } \\
\text { Spagnoli. }\end{array}$ & $\begin{array}{c}2015 \\
\text { Brasil } \\
\text { TEXTO CONTEXTO } \\
\text { ENFERM } \\
\text { Estudo transversal } \\
\text { descritivo quantitativo }\end{array}$ & $\begin{array}{l}\text { Objetivos: identificar os responsáveis pelos cuidados } \\
\text { com os produtos para saúde nas unidades de internação; } \\
\text { aspectos estruturais e recursos materiais disponíveis para } \\
\text { o seu transporte e armazenamento; descrever os cuidados } \\
\text { com o transporte e armazenamento de produtos para } \\
\text { saúde, e a ocorrência de eventos relacionados com esses } \\
\text { produtos. } 11 \text { unidades. Não atendidas recomendações } \\
\text { sobre estrutura, recursos materiais, transporte e } \\
\text { armazenamento dos produtos. Os eventos relacionados } \\
\text { revelaram descuidado da equipe de saúde nas unidades e } \\
\text { com risco para a manutenção da esterilidade dos produtos } \\
\text { e segurança do usuário. }\end{array}$ \\
\hline 13 & $\begin{array}{l}\text { "CUSTO DIRETO DO } \\
\text { REPROCESSAMENTO DE } \\
\text { CAMPOS CIRÚRGICOS DE } \\
\text { TECIDO DE ALGODÃO: UM } \\
\text { ESTUDO DE CASO” } \\
\text { Tomé \& Lima. }\end{array}$ & $\begin{array}{c}2015 \\
\text { Brasil } \\
\text { REV ESC ENFERM USP } \\
\text { Estudo de caso } \\
\text { quantitativo, exploratório- } \\
\text { descritivo }\end{array}$ & $\begin{array}{l}\text { Objetivo: identificar o custo direto do reprocessamento } \\
\text { de campos de tecido de algodão, duplos e simples, } \\
\text { integrantes do pacote de LAP cirúrgico. O custo total } \\
\text { médio, por pacote de LAP cirúrgico, foi de US\$ 9.72, } \\
\text { com predominância do custo com materiais (US\$ 8.70) } \\
\text { 89.65\%. Destaca-se que o custo total médio dos materiais } \\
\text { recebeu forte impacto dos custos dos campos de tecido de }\end{array}$ \\
\hline
\end{tabular}




\begin{tabular}{|c|c|c|c|}
\hline & & & algodão (US\$ 7.99) 91.90\%. \\
\hline 14 & $\begin{array}{l}\text { "MAPEAMENTO DO PROCESSO } \\
\text { DE REPROCESSAMENTO DE } \\
\text { CAMPOS CIRÚRGICOS DE } \\
\text { TECIDO DE ALGODÃO” } \\
\text { Tomé \& Lima. }\end{array}$ & $\begin{array}{c}2015 \\
\text { Brasil } \\
\text { REV. SOBECC } \\
\text { Estudo de caso exploratório } \\
\text { descritivo. }\end{array}$ & $\begin{array}{l}\text { Objetivo: mapear o processo de reprocessamento de } \\
\text { campos de tecido de algodão, duplos e simples, } \\
\text { integrantes dos pacotes de LAP cirúrgico. O mapeamento } \\
\text { das etapas envolvendo a lavanderia e a CME possibilitou } \\
\text { a visualização dos recursos consumidos e atividades } \\
\text { constituintes do processo. }\end{array}$ \\
\hline 15 & $\begin{array}{l}\text { "ARMAZENAMENTO DOS } \\
\text { PRODUTOS PARA SAÚDE } \\
\text { ESTÉREIS EM UNIDADES } \\
\text { ASSISTENCIAIS: ESTUDO } \\
\text { DESCRITIVO" } \\
\text { Oliveira, Mussel, \& Paula. }\end{array}$ & $\begin{array}{c}2014 \\
\text { Brasil } \\
\text { REV. SOBECC } \\
\text { Estudo exploratório, } \\
\text { descritivo }\end{array}$ & $\begin{array}{l}\text { Objetivo: analisar as condições dos locais de guarda dos } \\
\text { produtos para saúde estéreis em unidades assistenciais de } \\
\text { hospitais de grande porte de Belo Horizonte, Minas } \\
\text { Gerais. Locais de acondicionamento do produto de } \\
\text { revestimento lavável }(93,9 \%) \text {; armazenados em armários } \\
\text { fechados }(75,8 \%) \text {; validade da esterilização observada } \\
(97 \%) \text {. Foram encontradas áreas não exclusivas para } \\
\text { guarda de artigos esterilizados. }\end{array}$ \\
\hline
\end{tabular}

Fonte: Autores (2021).

Os estudos selecionados foram predominantemente brasileiros (66,7\%). Os demais eram provenientes da China, Indonésia, Índia e Estados Unidos. O ano de 2015 foi o mais prevalente (33,3\%). Estudos descritivos representaram 53,3\% do total, seguindo-se os experimentais $(20 \%)$.

\section{Discussão}

A análise da área de armazenamento de produtos estéreis foi realizada em três cenários distintos: a CME, uma clínica odontológica e unidades de internação. Neles, uma série de problemas ou não conformidades foi evidenciada nos estudos.

A frequência de descontaminação dessa área limpa (Oliveira, Mussel, \& Paula, 2014), condições de temperatura e umidade fora dos padrões nacionais e internacionais recomendados (Nunes et al., 2019), ou mesmo a falta de controle desses parâmetros foram revelados (Maciel, Martins, Mendes, Kozusny-Andreani, \& de Souza, 2019).

Ainda em termos das condições ambientais da área de armazenamento foram citados a incidência da luz solar direta e tubulações expostas (Mussel, de Paula, \& de Oliveira, 2017). Estudo apontou que em 30\% dos hospitais não havia área exclusiva para produtos estéreis (Mussel et al., 2017). Nas unidades de internação, pela falta de norma regulamentadora para o armazenamento de produtos estéreis, estes eram armazenados de maneira inadequada, em corredores, dando total acesso a qualquer pessoa do hospital (Freitas, Tipple, Pires, Melo, \& Spagnoli, 2015). Em setores como centros de terapia intensiva, centros cirúrgicos e pronto atendimentos o armazenamento de produtos estéreis ocorria em armários apertados e gavetas, podendo haver a violação dos invólucros, como também produtos estéreis que se encontravam próximos a produtos não estéreis, gerando o risco de contaminação (Oliveira et al., 2014). Vale mencionar que até mesmo a limpeza da área de armazenamento se reveste de cuidado especializado. Em estudo sobre a descontaminação das prateleiras na área de armazenamento, constataram-se eventos adversos, como a queda dos produtos estéreis, o rompimento do sistema de barreira por deslocar os produtos pela fricção nas prateleiras (Tipple, dos Santos, de Melo Costa, Bouwman, \& Goulart, 2020). O condicionamento de ar foi mencionado como causa provável para contaminação dos produtos (Kurniawansyah, Abdassah, \& Gondodiputro, 2015).

A possibilidade ou mesmo a confirmação da contaminação de produtos estéreis foi constatada por diferentes razões. Entre elas podem ser citadas: toque excessivo no sistema de barreira, manuseio incorreto do produto, falta de higiene das mãos (Tipple et al., 2020), tempo de permanência de 84 dias em prateleira em área de armazenamento sem controle de temperatura e umidade (Maciel et al., 2019). 
Os problemas ambientais foram apresentados como possíveis fatores para contaminação de produtos estéreis, gerando a demanda de novo processamento e consumo de sistemas de barreira estéril. Além deles, falhas em outras fases do processamento foram encontradas. Produtos vencidos, resíduos orgânicos pós- esterilização (Alvim \& de Souza, 2018), materiais limpos e sujos no setor de armazenamento (Oliveira et al., 2014), especificação errada, falta de instrumentos, pacote incompleto (Zhu, Yuan, Li \& Cheng, 2019), todos esses elementos provocam a necessidade de um novo processamento, aumentando o consumo de sistemas de barreira estéril.

O custo pelo consumo dos sistemas de barreira estéril foi estimado para o reprocessamento de campos de tecido de algodão integrantes do pacote de laparotomia (LAP) cirúrgico, atingindo o percentual de 91,90\% dos custos totais (Tomé \& Lima, 2015a; Tomé \& Lima, 2015b). Esse elevado percentual demanda outras análises sobre custo e benefício com outros sistemas de barreira estéril, os descartáveis.

O não tecido, um sistema de barreira descartável foi avaliado em termos da relação custo-benefício e os resultados após a introdução do seu uso e a ocorrência da resterilização. Os resultados apontaram uma redução na taxa de resterilização de 9 para 0,5\% em um período de 4 anos. Cabe ressaltar o aumento na geração de resíduos sólidos produzido pelos sistemas de barreira estéril descartáveis. Por outro lado, a preocupação com o meio ambiente, o consumo de água e energia não podem ser esquecidos (Devadiga, Thomas, Shetty, \& Setia, 2015).

As descontinuidades no sistema de barreira estéril, furos ou orifícios e a percepção dos mesmos pelos profissionais foram alvo de um estudo. Os resultados sinalizaram que orifícios menores que $2 \mathrm{~mm}$ não foram detectados em um grupo de 30 profissionais. O tempo de experiência profissional, o tipo de iluminação e o tempo de inspeção não interferiram nos resultados (Rashidifard et al., 2018).

O consumo de sistemas de barreira estéril além do previsto, com base nos estudos analisados, se explica em função de uma série de falhas em diferentes fases do processamento tais como o acondicionamento, a inspeção, além do armazenamento em si (Oliveira et al., 2014; Kurniawansyah et al., 2015; Mussel et al., 2017; Nunes et al., 2019; Zhu et al., 2019; Tipple et al., 2020).

Outros elementos relativos ao fluxo e condições de trabalho na CME surgiram como resultado de alguns estudos, embora não se constituíssem no objetivo primário dos mesmos. Aumento da taxa de erros em pacotes no horário entre 16 e 22 horas (Zhu et al., 2019), 605 diferentes causas de retrabalho na CME (Alvim \& de Souza, 2018) permitem inferir sobre o cansaço dos profissionais da CME e a necessidade de melhorias nas condições de trabalho reduzindo a possibilidade de falhas nessa unidade.

O problema relativo ao armazenamento de produtos estéreis, consumo excessivo de sistemas de barreira estéril, resterilizações e retrabalho foi delineado como um problema sem fronteiras, constatado em outros países (Kurniawansyah et al., 2015; Kurniawansyah \& Mita, 2017).

Para o nosso país, muitos déficits foram mencionados como a falta de insumos e equipamentos de qualidade como: invólucros, seladoras, ultrassônicas (Reisdorfer, de Araujo, da Silva, dos Santos, \& Soder, 2016; Alvim \& de Souza, 2018). Por outro lado, o processamento de produtos para a saúde estéril não utilizados foi apontado como importante fator para o retrabalho dos profissionais nas CMEs (Alvim \& de Souza, 2018). Maior o consumo de sistemas de barreira estéril, maior a quantidade de resíduos gerada. Mais carga de trabalho para a CME. As unidades de saúde no seu dia a dia já consomem muita energia, água e geram grande quantidade de resíduos. Qualquer desperdício é totalmente desnecessário.

A educação continuada foi sugerida como estratégia para atualizar os profissionais, buscando a padronização dos serviços nas CMEs, de acordo com as normas vigentes (Reisdorfer et al., 2016). 


\section{Conclusão}

As publicações selecionadas sobre o armazenamento de produtos estéreis permitem concluir que se trata de fase do processamento ainda com muitos desafios não somente em nosso país, mas em parte do mundo.

As condições ambientais da área de armazenamento precisam ser aprimoradas em termos do controle da temperatura, umidade e descontaminação do local.

O consumo de sistemas de barreira estéril aumenta não somente em função das condições de armazenamento. Estudos evidenciaram uma série de problemas que resultaram em novo processamento, uma nova esterilização e retrabalho para a equipe da CME. Entre essas falhas podem ser citados: pacotes incompletos, produtos vencidos, resíduos orgânicos pósesterilização; materiais limpos e sujos no setor de armazenamento, especificação errada, falta de instrumentos.

Os sistemas de barreira estéril foram analisados sob diferentes óticas como o custo benefício e a integridade. A não percepção de orifícios menores que $2 \mathrm{~mm}$ independentemente do tipo de iluminação ou experiência profissional foi confirmada em um estudo.

O impacto do trabalho e retrabalho da CME sobre a sustentabilidade do meio ambiente é grande. Nessa unidade ocorre um gasto de água e energia consideráveis. A definição sobre os sistemas de barreira estéril (SBE) deverá contemplar a geração de resíduos pelos descartáveis e também a complexo processamento do tecido de algodão que envolve a CME, a lavanderia entre outros setores.

Destacam-se ainda as infrações sanitárias evidenciadas nos estudos. Maior atenção na qualidade do armazenamento do produto para saúde estéril é requerida a fim de preservar sua condição de esterilidade, assegurando o uso seguro.

Recomenda-se para trabalhos futuros, artigos que busquem expandir a sustentabilidade no Centro de Material e Esterilização, de forma que possa ser dada atenção para a redução de danos ambientais, sociais e econômicos.

Por fim, sugere-se também a necessidade de educação continuada permitindo a revisão de práticas incorretas como a não higienização das mãos antes do manuseio de produtos estéreis e o conhecimento de recomendações dos órgãos regulamentadores que tem que ser atendidas.

\section{Referências}

Alvim, A. L. S., \& de Souza, K. F. (2018). Causas de retrabalho de produtos para Saúde no centro de materiais e esterilização. Rev. SOBECC, 3-6. $10.5327 / \mathrm{Z} 1414-4425201800010002$

AORN, Conner, R., \& Blanchard, J. C. (2017). Perioperative standards and recommended practices. AORN.

Brasil. (2012). RDC n ${ }^{\circ}$ 15, de 15 Mar 2012. Dispõe sobre requisitos de boas práticas para o processamento de produtos para saúde e dá outras providências. https://bvsms.saude.gov.br/bvs/saudelegis/anvisa/2012/rdc0015_15_03_2012.html

Devadiga, G. S., Thomas, V. M. P., Shetty, S., \& Setia, M. S. (2015). Is non-woven fabric a useful method of packaging instruments for operation theatres in resource constrained settings? Indian journal of medical microbiology, 33(2), 243-247. 10.4103/0255-0857.154862

Freitas, L. R. D., Tipple, A. F. V., Pires, F. V., Melo, D. D. S., \& Spagnoli, J. L. U. (2015). (Des) cuidado com produtos para saúde processados no transporte e armazenamento em unidades de internação1. Texto \& Contexto-Enfermagem, 24, 253-262. http://dx.doi.org/10.1590/0104-07072015003550013

Galvão, T. F., Pansani, T. D. S. A., \& Harrad, D. (2015). Principais itens para relatar Revisões sistemáticas e Meta-análises: A recomendação PRISMA. Epidemiologia e Serviços de Saúde, 24, 335-342.

Joanna Briggs Institute. (2014). Joanna Briggs Institute reviewers’ manual: 2014 edition. Australia: The Joanna Briggs Institute.

Justiniano, G. P. D. M., Eduardo, A. H. A., Binotto, C. C. S., Macedo, J. I., Veiga, T. B., Tognoli, S. H., \& Mendes, A. A. (2020). Riscos ocupacionais e os resíduos de serviços de saúde em centro cirúrgico. Rev. SOBECC, 25-32.

Kurniawansyah, I. S., Abdassah, M., \& Gondodiputro, S. (2015). Relationship between temperature and humidity on sterility of reusable instruments in hospital's CSSD. Int. J. Pharm. Sci. Rev. Res, 33(2), 215-219.

Kurniawansyah, I. S., \& Mita, S. R. (2017). The Sterility of Reusable Instruments at Central Surgery Installation's Room Storage of One of The Hospital in Bandung. Journal of Pharmaceutical Sciences and Research, 9(6), 936. 
Research, Society and Development, v. 10, n. 9, e36010918201, 2021

(CC BY 4.0) | ISSN 2525-3409 | DOI: http://dx.doi.org/10.33448/rsd-v10i9.18201

Maciel, Y. K. Q., Martins, C. C., Mendes, E. C. B., Kozusny-Andreani, D. I., \& de Souza, T. F. Q. (2019). Prazo de validade de esterilização de artigos utilizados em uma clínica odontológica. Nursing (São Paulo), 22(250), 2794-2799.

Mussel, I. C., de Paula, A. O., \& de Oliveira, A. C. (2017). Armazenamento dos produtos para saúde em centros de esterilização de hospitais. Enfermagem em Foco, 8(4).

Nogueira, D. N. G., Santos, G. A., Cardelli, A. A. M., \& Castilho, V. (2020). Resíduos de serviços de saúde: perfil e análise de custos em um centro cirúrgico. Rev. SOBECC, 151-158.

Nunes, M. B. de S., Fassarella, C. S., Souza, P. A. de, Meneses, R. de O., Silva, M. V. G. da, \& Luna, A. A. (2019). Condições de temperatura e umidade na sala de distribuição e armazenamento dos produtos para saúde. Revista Enfermagem Atual In Derme, 87(25). https://doi.org/10.31011/reaid-2019-v.87n.especial-art. 260

Oliveira, A. C., \& Silva, M. V. G. (2015). Teoria e prática na prevenção da infecção do sítio cirúrgico. Manole.

Oliveira, A. C. D., Mussel, I. C., \& Paula, A. O. (2014). Armazenamento dos produtos para saúde estéreis em unidades assistenciais: estudo descritivo. Rev. SOBECC, 188-194. 10.5327/Z1414-4425201400040003

Oliveira, E. L., Viana, V. J., \& Castañon, A. B. (2018). Performance Ambiental em Estabelecimentos de Saúde: Um Estudo de Caso do Hospital Naval Marcílio Dias, Rio de Janeiro-RJ. Revista de Gestão Ambiental e Sustentabilidade, 7(3), 520-538. https://doi.org/10.5585/geas.v7i3.968

Pereira, R. R. N., Pascoal, L. M., Rolim, I. L. T. P., Ferreira, A. G. N., \& Silva, E. L. D. (2020). Custo de processamento de produtos para saúde: uma revisão integrativa. Rev. SOBECC, 105-113.

Rashidifard, C. H., Mayassi, H. A., Bush, C. M., Opalacz, B. M., Richardson, M. W., Muccino, P. M., \& DiPasquale, T. G. (2018). Looking for holes in sterile wrapping: How accurate are we?. Clinical orthopaedics and related research, 476(5), 1076. DOI 10.1007/s11999.0000000000000185

Reisdorfer, N., de Araujo, G. M., da Silva, L. A. A., dos Santos, A. M., \& Soder, R. M. (2016). < b> Segurança do paciente: embalagens, acondicionamento e tempo de guarda de materiais esterilizados na Atenção Básica/Safety: packages, packing and storage period of sterilized materials in Primary Care < b. Ciência, Cuidado E Saúde, 15(4), 662-668. 10.4025/cienccuidsaude.v15i4.29925

SOBECC, S. P. R. (2013). Sociedade Brasileira de Enfermeiros de Centro Cirúrgico. Recuperação Anestésica e Centro de Material e Esterilização. SOBECC.

Tipple, A. F. V., dos Santos, J. E. M., de Melo Costa, D., Bouwman, B. E., \& Goulart, D. M. M. (2020). < b> Área de armazenamento de produtos para saúde: repensando a frequência da descontaminação de prateleiras/Reusable medical devices storage area: rethinking the shelves decontamination frequency. Ciência, Cuidado e Saúde, 19. 10.4025/cienccuidsaude.v19i0.48223

Tomé, M. F., \& Lima, A. F. C. (2015a). Custo direto do reprocessamento de campos cirúrgicos de tecido de algodão: um estudo de caso. Revista da Escola de Enfermagem da USP, 49, 488-494. 10.1590/S0080-623420150000300018

Tomé, M. F., \& Lima, A. F. C. (2015b). Mapeamento do processo de reprocessamento de campos cirúrgicos de tecido de algodão. Rev SOBECC 2015a out/dez, 20(4), 197-201. 10.5327/Z1414-4425201500040003

Zhu, X., Yuan, L., Li, T., \& Cheng, P. (2019). Errors in packaging surgical instruments based on a surgical instrument tracking system: an observational study. BMC health services research, 19(1), 1-6. https://doi.org/10.1186/s12913-019-4007-3 